\title{
Erratum to: Nanomaterials in Medicine
}

\author{
Zhypargul Abdullaeva
}

\section{Erratum to: \\ Chapter 4 in: Z. Abdullaeva, Nanomaterials in Daily Life, DOI 10.1007/978-3-319-57216-1}

In the original version of the book, a new reference and its citation have to be included in Chapter 4. The erratum chapter and the book have been updated with the change. 